\title{
Structure and Haemocompatibility of Tetrahedral Amorphous Carbon Films Prepared by Pulsed Laser Ablation
}

\author{
Min Chen, Zhanyun Huang, Lin Zhang, Dihu Chen* and Aixiang Wei ${ }^{1}$ \\ State Key Laboratory of Optoelectronic Materials and Technologies, and School of Physics \& \\ Engineering, Sun Yat-Sen University, Guangzhou 510275, P. R. China \\ ${ }^{1}$ Faculty of Material and Energy, Guangdong University of Technology, \\ Guangzhou 510006, P. R. China
}

(Received July 22, 2008; accepted November 25, 2008)

Key words: tetrahedral carbon films, Raman spectrum, haemocompatibility, pulsed laser deposition

Tetrahedral amorphous carbon films (ta-C) as promising blood-contacting biomaterials have been prepared by pulsed laser deposition. The structural and optical properties of these films have been studied by scanning electron microscopy, Raman spectroscopy, UV-visible optical absorption spectroscopy and contact angle measurement. Results show that ta-C films with different bond ratios of $\mathrm{sp}^{3} \mathrm{C}$ to $\mathrm{sp}^{2} \mathrm{C}$ can be obtained by changing the repetition frequency of the pulsed laser during deposition. The blood compatibility of the samples was evaluated by tests of platelet adhesion and kinetic clotting time. The quantity and morphology of the adherent platelets on the surface of samples were investigated by scanning electron microscopy. The haemocompatibility of ta-C films depends on the bond ratio of $\mathrm{sp}^{3} \mathrm{C}$ to $\mathrm{sp}^{2} \mathrm{C}$ and the better blood compatibility of the samples is attributed to a suitable $\mathrm{sp}^{3} \mathrm{C}$ fraction in ta-C films.

\section{Introduction}

The high hardness, low coefficient of friction, chemical inertness, and excellent surface finish of diamond-like carbon (DLC) make it attractive as a coating in biomedical implants, such as joints, orthopedic pins and screws, dental prostheses, and medical

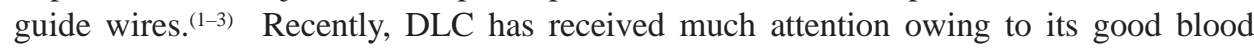
compatibility, and it has been suggested as a potential biomaterial in blood-contacting devices. $^{(4-6)}$ However, DLC films often contain a significant hydrogen concentration (up to and exceeding 10\%). Hydrogen terminates many structural bonds and reduces hardness. In contrast, tetrahedral amorphous carbon (ta-C) films are typical hydrogenfree $(<0.1 \%)$ carbon films. Therefore, these films can exhibit a higher hardness (up to 9/10 that of natural diamond). However, relatively little is known about the

*Corresponding author: e-mail: stscdh@mail.sysu.edu.cn 
biocompatibility of ta-C films. ${ }^{(6-8)}$ Pulsed laser deposition (PLD) has proved to be an effective technique for the deposition of a wide variety of thin film materials. ${ }^{(9)}$ Researchers $^{(10)}$ have studied the biocompatibility of ta-C films deposited by pulsed laser ablation. However, none of these studies consider the wettability and how to modulate the bond ratio of $\mathrm{sp}^{3} \mathrm{C}$ to $\mathrm{sp}^{2} \mathrm{C}$ in ta-C films, which play an important role in blood compatibility. In this work, ta-C films were produced by PLD, and the structure, wettability, and haemocompatibility of ta-C films were investigated.

\section{Materials and Methods}

The ta-C films were deposited on single-crystal silicon (100) and quartz substrates by PLD. A KrF excimer laser (Thin Filmstar TM V2.0, Germany) operating at a frequency of $248 \mathrm{~nm}$ was used for the ablation of the graphite target. The laser was operated at a pulsed duration of $20 \mathrm{~ns}$ and the repetition rate was varied from 10 to $90 \mathrm{~Hz}$. Laser pulses provided an average energy density of about $2 \mathrm{~J} / \mathrm{cm}^{2}$ to the target. The target material was graphite with a purity of $99.99 \%$. The substrate was mounted on a metal substrate stage holder parallel to the target at a distance of about $70 \mathrm{~mm}$. The deposition chamber was evacuated to a base pressure of approximately $2 \times 10^{-5}$ Torr using a turbomolecular pump. To ensure a uniform ablation rate, the target was rotated at $5 \mathrm{rpm}$ during deposition, and the total time for each deposition was 10 min. Raman spectra were obtained using a Raman system (Renishaw 2000) with a laser source at $514 \mathrm{~nm}$. The Raman spectra were fitted using a single skewed Breit-Wigner-Fano (BWF) lineshape and a doubleLorentzian lineshape for the samples with large and small fractions of $\mathrm{sp}^{3}$ carbon bonding, respectively. The BWF coupling coefficient $\mathrm{Q}$, which is highly sensitive to the $\mathrm{sp}^{3}$ fraction of the films, was used to estimate the $\mathrm{sp}^{3}$ fraction of the samples with larger $\mathrm{sp}^{3}$ fractions. The optical absorption measurements of ta-C films were carried out using a double-beam UV-visible spectrometer with a bare quartz substrate in the reference beam to eliminate the substrate contribution. The surface energy and interfacial tension of the samples were determined by contact angle measurements in accordance with the description in ref. 11. Distilled water and glycol were used in our tests to determine the surface energy and interfacial tension of the samples. Measurements of the platelet adhesion and kinetic clotting time were performed to identify the blood compatibility of ta-C films. These blood compatibility measurements were performed in accordance with the standard procedure of the authority department. In all tests, ChronoFlex was used as a negative reference in clinical application, and glass was used as a positive reference, where negative and positive reference samples were defined as samples with better and worse haemocompatibilities, respectively. The quantity, morphology, aggregation, and pseudopodium of the adherent platelets on the surfaces of the samples were observed by scanning electron microscopy (SEM), and ten different regions were randomly chosen for each sample to obtain good statistics. For the kinetic clotting time measurement, the concentration of hemoglobin was identified by the absorbency of the solution, which was detected using a spectrophotometer at $540 \mathrm{~nm}$. In our experiments, the kinetic clotting time was defined as the time interval when the absorbency of the solution is 0.1 . 


\section{Results and Discussion}

Raman spectra of ta-C films deposited on silicon substrates at different repetition frequencies from 10 to $90 \mathrm{~Hz}$ were investigated. The spectra exhibit a broad Raman intensity distribution in the range $1200-1700 \mathrm{~cm}^{-1}$ centered at $1570 \mathrm{~cm}^{-1}$ and a square $\mathrm{Si}$ band at $950 \mathrm{~cm}^{-1}$. The latter is due to the second-order Raman spectrum of the underlying Si substrate. As shown in Fig. 1, the Raman spectra show a repetition frequency dependence. For the films deposited at a repetition frequency of $30 \mathrm{~Hz}$ or above $50 \mathrm{~Hz}$, the spectra show a broad asymmetric Raman intensity distribution, and a reasonable fitting to data could be achieved using two Lorentzian peaks at $1570 \mathrm{~cm}^{-1}$ (G peak) and $1370 \mathrm{~cm}^{-1}$ (D peak). The ratio of integrated intensity, $I_{\mathrm{D}} / I_{\mathrm{G}}$, and the $\mathrm{G}$ peak position as a function of the repetition frequency are respectively plotted in Figs. 2(a) and 2(b), which give information on the bond ratio of $\mathrm{sp}^{3} \mathrm{C}$ to $\mathrm{sp}^{2} \mathrm{C}$ or the $\mathrm{sp}^{3} \mathrm{C}$ fraction in ta-C films. Results show that the $I_{\mathrm{D}} / I_{\mathrm{G}}$ ratio decreases with increasing repetition frequency from 10 to $50 \mathrm{~Hz}$ and then increases with a repetition frequency increase from 50 to $90 \mathrm{~Hz}$. A lower $I_{\mathrm{D}} / I_{\mathrm{G}}$ ratio corresponds to a higher $\mathrm{sp}^{3} \mathrm{C}$ fraction in ta-C films. When the repetition frequency is above $50 \mathrm{~Hz}$, the increase in the $I_{\mathrm{D}} / I_{\mathrm{G}}$ ratio indicates the presence of a larger number of $\mathrm{sp}^{2}$ carbon bonds or of small graphite crystallites in the films. To estimate the $\mathrm{sp}^{3}$ fraction $\left[\mathrm{sp}^{3}\right] /\left(\left[\mathrm{sp}^{2}\right]+\left[\mathrm{sp}^{3}\right]\right)$ in these films by Raman spectroscopy, we used the method mentioned in ref. 12 to fit the spectra with a single skewed BWF lineshape described by

$$
I(\omega)=I_{0}\left(1+2\left(\omega-\omega_{0}\right) / Q \Gamma\right)^{2} /\left\{1+\left[2\left(\omega-\omega_{0}\right) / \Gamma\right]^{2}\right\},
$$

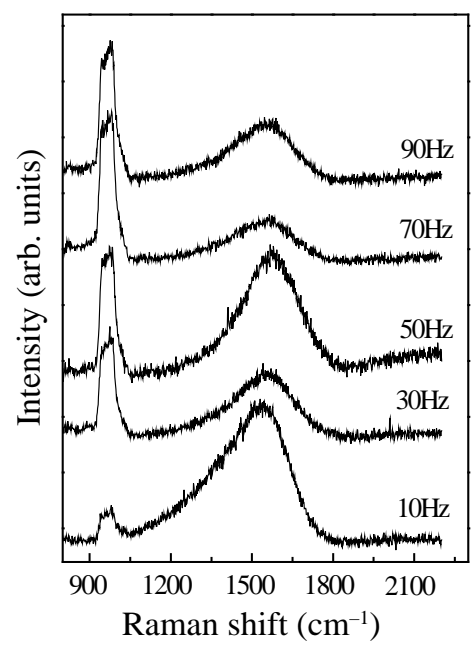

Fig. 1. Raman spectra of ta-C films prepared at different repetition frequencies of the pulsed laser. 


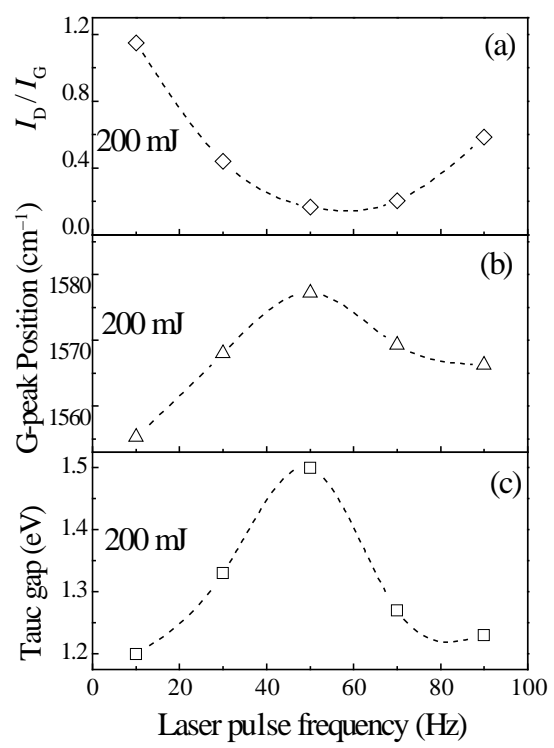

Fig. 2. Repetition frequency dependence of (a) intensity ratio of D Raman peak to G Raman peak $I_{\mathrm{D}} / I_{\mathrm{G}}$; (b) G-band position of Raman spectra; and (c) optical gap $E_{g}$ determined from Tauc relationship.

where $I(\omega)$ is the intensity as a function of frequency, $I_{0}$ is the maximum peak intensity, $\omega_{0}$ is the peak position, $\Gamma$ is the full width at half maximum (FWHM), and $Q$ is the BWF coupling coefficient. As shown in ref. 12, the BWF coupling coefficient $Q$ is highly sensitive to the $\mathrm{sp}^{3}$ fraction of the films. The films with larger $\mathrm{sp}^{3}$ fractions have much more symmetrical bands and $|Q|\left(Q\right.$ is negative) increases as the $\mathrm{sp}^{3}$ fraction increases. In our experiments, the spectra of these ta-C films could be fitted using a single BWF lineshape. The coupling coefficients of the films were determined to be about $-18,-22$, $-26,-23$, and -20 , corresponding to the samples deposited at the repetition frequencies of the pulsed laser of 10, 30, 50, 70, and $90 \mathrm{~Hz}$, respectively. According to the analysis method in ref. 12 , the $\mathrm{sp}^{3}$ fraction is probably more than $80 \%$ if $Q$ is less than about -20 . Thus, we found that the $\mathrm{sp}^{3}$ fraction of most ta-C films deposited by laser ablation is greater than $80 \%$, showing that the sample deposited at $50 \mathrm{~Hz}$ has a maximum $|Q|$ value, indicating that the $\mathrm{sp}^{3}$ fraction of this sample is about $90 \%$. The estimation maybe determined using other methods, such as electron energy-loss spectroscopy (EELS) ${ }^{(13)}$ and X-ray photoelectron spectroscopy (XPS). ${ }^{(14)}$

The optical band gaps of these ta-C films determined by the Tauc relationship $\alpha E$ $=B\left(E-E_{\mathrm{g}}\right)^{2}$ are shown in Fig. 2(c). It was found that the optical band gap increases with increasing repetition frequency of the pulsed laser during deposition and reaches a maximum value of about $1.5 \mathrm{eV}$ at $50 \mathrm{~Hz}$, and then continuously decreases with increasing repetition frequency. Hauser ${ }^{(15)}$ suggested that ta- $\mathrm{C}$ films contain a mixture of 
diamond ( $\mathrm{sp}^{3} \mathrm{C}$ bond) and graphite ( $\mathrm{sp}^{2} \mathrm{C}$ bond) bonds. Therefore, the optical band gap of ta-C films depends on the hybridized $\mathrm{C}$ bond ratio of $\mathrm{sp}^{3}$ to $\mathrm{sp}^{2}$. The smaller optical gap indicates that the optical absorption in these samples is mainly caused by the $\pi$ states and the $\mathrm{sp}^{2} \mathrm{C}$ fraction of these samples is larger than that of the other samples.

Typical SEM images of platelet adhesion for ta-C films prepared at various repetition frequencies of the pulsed laser are shown in Fig. 3. Results show that there is less platelet aggregation and pseudopodium on ta-C films (Figs. 3(a)-3(d)), even less than that on the negative reference sample (ChronoFlex) (Fig. 3(e)), thus exhibiting better haemocompatibility. Platelet aggregation was clearly observed for the positive reference sample (glass), as shown in Fig. 3(f). The number of platelets adhering to the surfaces of samples deposited at different repetition frequencies was counted, and the results are presented in Fig. 4(a). The results show that the number of platelets adhering to the surface of the sample prepared at $50 \mathrm{~Hz}$ is less than that in the cases of the negative reference sample (ChronoFlex) and the positive reference sample (glass). Typically, the
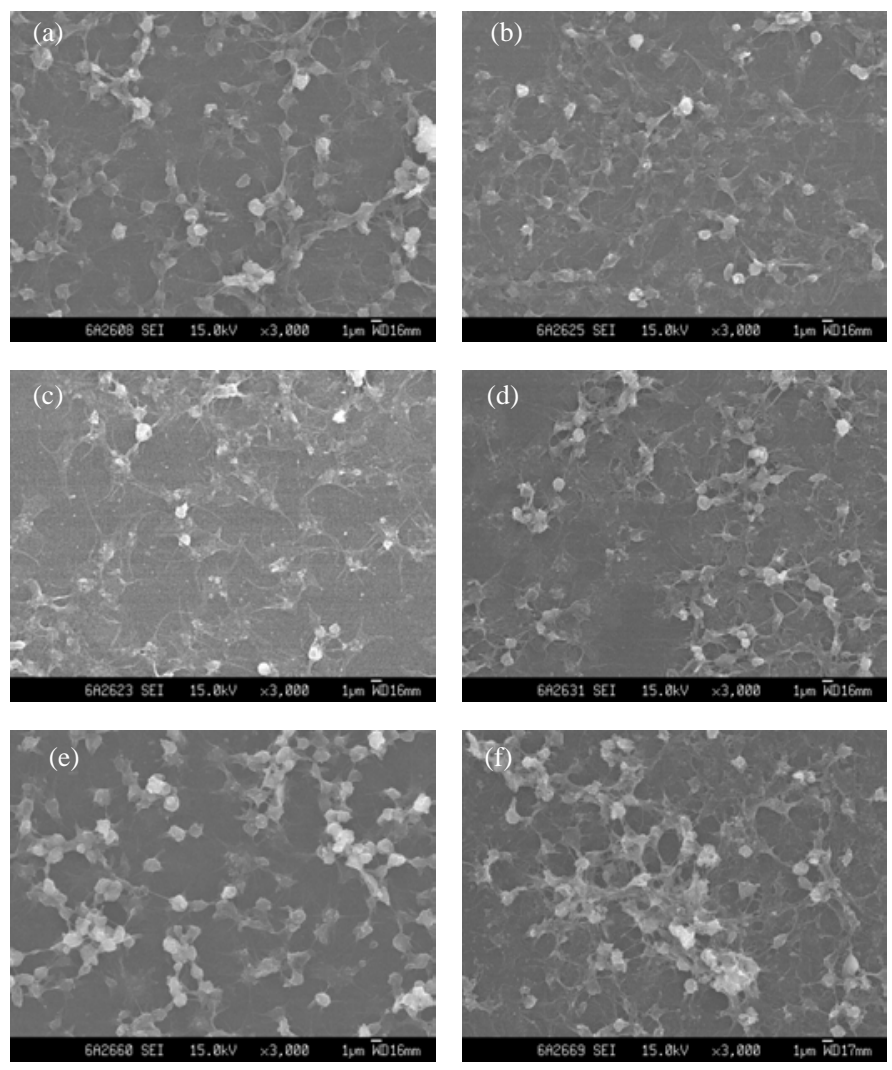

Fig. 3. Typical SEM images of platelets adhering to surfaces of ta-C films prepared at repetition frequencies of (a) $10 \mathrm{~Hz}$; (b) $30 \mathrm{~Hz}$; (c) $50 \mathrm{~Hz}$; and (d) $70 \mathrm{~Hz}$; and on (e) ChronoFlex and (f) glass. 


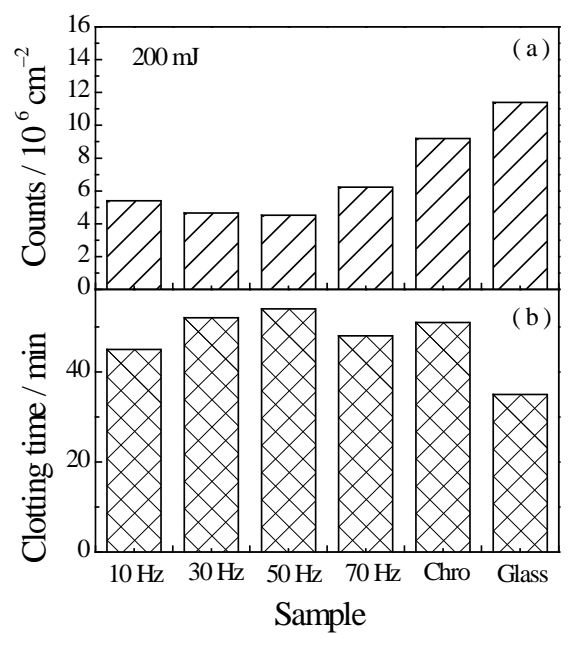

Fig. 4. (a) Number of platelets adhering to surfaces of ta-C films deposited at various repetition frequencies and on negative (ChronoFlex) and positive (glass) references; (b) kinetic clotting time for these samples.

less the platelet adheres to the surface of the sample, the better its haemocompatibility. Therefore, the sample prepared at $50 \mathrm{~Hz}$ may exhibit better haemocompatibility. Results of the kinetic clotting time measurement of the samples are shown in Fig. 4(b). In general, the longer the kinetic clotting time of the sample, the better its haemocompatibility. As shown in Fig. 4(b), the kinetic clotting time is observed to be different for the samples prepared at various repetition frequencies. The kinetic clotting times of the samples prepared at 30 and $50 \mathrm{~Hz}$ are approximate and the longest kinetic clotting time is that for these two samples, which is even longer than that of the negative reference sample, indicating that these two samples have better anticruor properties.

The interaction between blood and contacting biomaterials is very complex and the detailed mechanism of haemocompatibility of ta-C films with various bond ratios of $\mathrm{sp}^{3}$ $\mathrm{C}$ to $\mathrm{sp}^{2} \mathrm{C}$ is still unclear. Baurschmidt and Schaldach ${ }^{(16)}$ reported that the formation of thrombus on the biomaterial surface is correlated with charge transfer from fibrinogen to the material surface. Fibrinogen can transform to fibrin monomer and fibrinopeptides when it loses charge. The cross-link of fibrin monomer causes irreversible thrombus formation. Thus, a suitable charge density promotes haemocompatibility. A suitable bond ratio of $\mathrm{sp}^{3} \mathrm{C}$ to $\mathrm{sp}^{2} \mathrm{C}$ can provide an optimum charge density to promote haemocompatibility. Results obtained from various measurement methods exhibited the same tendency, and the ta-C films prepared at $50 \mathrm{~Hz}$ were considered to be the best in terms of haemocompatibility properties among this batch of samples. Thrombus formation involves a complex cascade of biochemical reactions and path signaling biological processes, ${ }^{(17)}$ with the biochemical, electrochemical, and physical behaviors 
of individual blood components, such as coagulation factors, thrombocytes, and selected enzymes coming into contact with a solid-body surface. The properties of the biomaterial surface, such as surface energy, roughness, surface hydrophobicity, and zeta potential, affect the haemocompatibility of the biomaterial, ${ }^{(18,19)}$ but it is difficult to demonstrate the effect of each factor. The initial step after blood comes in contact with the biomaterial is the formation of an adhered layer of protein, which determines the anticoagulation property of the biomaterial.(20,21) Two primary factors related to thrombus formation are the adsorptions of human fibrinogen (HFG) and human serum albumin (HSA). It has been proven that the adsorption of HFG promotes the adhesion of platelets and activates the platelets, whereas the adsorption of HSA does not.(22) The protein adsorption on the surfaces of biomaterials is related to the surface energy. ${ }^{(23)}$ In this work, the contact angle, surface free energy components, and interfacial tension of ta-C films are summarized in Table 1. Results show that the ta-C films have similar contact angles, surface free energy components, and interfacial tensions. Our studies suggest that ta-C films with various bond ratios of $\mathrm{sp}^{3} \mathrm{C}$ to $\mathrm{sp}^{2} \mathrm{C}$ can be obtained by adjusting the repetition frequency during deposition, resulting in the different blood compatibilities of the samples. The interaction between blood and artificial materials is very complex and the detailed mechanism of haemocompatibility of ta-C films with various bond ratios of $\mathrm{sp}^{3} \mathrm{C}$ to $\mathrm{sp}^{2} \mathrm{C}$ is not clear yet. More work is required before the exact enhancement mechanism can be elucidated, particularly in evaluating the protein adsorption on the material surfaces.

\section{Conclusions}

Tetrahedral amorphous carbon films with different $\mathrm{sp}^{3}$ C-bond fractions were obtained by changing the repetition frequency of the pulsed laser during deposition. The haemocompatibility of ta-C films was investigated by platelet adhesion experiments and kinetic clotting time measurements. Results show that the haemocompatibility of ta-C films varies with the $\mathrm{sp}^{3} \mathrm{C}$ fraction of the sample. The haemocompatibility of ta-C films depends on the $\mathrm{sp}^{3} \mathrm{C}$ fraction and the better blood compatibility of the samples is attributed to the suitable $\mathrm{sp}^{3} \mathrm{C}$ fraction in ta-C films.

Table 1

Contact angle, surface energy components, and interfacial tension towards distilled water.

\begin{tabular}{cccccc}
\hline \multirow{2}{*}{ Samples } & $\begin{array}{c}\text { Contact angle } \\
\text { (degrees) }\end{array}$ & \multicolumn{3}{c}{$\begin{array}{c}\text { Surface energy } \\
\text { (dyn/cm) }\end{array}$} & $\begin{array}{c}\text { Interfacial tension } \\
\text { (dyn/cm) }\end{array}$ \\
\cline { 3 - 6 } & & \multicolumn{1}{c}{$\gamma_{s}$} & $\gamma_{s}{ }^{d}$ & \multicolumn{1}{c}{$\gamma_{s}{ }^{p}$} & $\gamma_{s l}$ \\
\hline $200 \mathrm{~mJ}-10 \mathrm{~Hz}$ & 73.63 & 33.37 & 20.7 & 12.67 & 53.89 \\
$200 \mathrm{~mJ}-30 \mathrm{~Hz}$ & 75.32 & 33.29 & 22.47 & 10.82 & 51.74 \\
$200 \mathrm{~mJ}-50 \mathrm{~Hz}$ & 77.47 & 33.05 & 24.11 & 8.94 & 48.84 \\
$200 \mathrm{~mJ}-70 \mathrm{~Hz}$ & 75.43 & 32.62 & 21.99 & 10.63 & 50.94 \\
$200 \mathrm{~mJ}-90 \mathrm{~Hz}$ & 81.33 & 30.86 & 23.62 & 7.24 & 41.83 \\
\hline
\end{tabular}




\section{Acknowledgment}

This work was supported in part by the National Natural Science Foundation of China, NSFC30770588 and 30370410.

\section{References}

1 M. Allen, B. Myer and N. Rushton: J. Biomed. Mater. Res. 58 (2001) 319.

2 M. I. Jones, I. R. McColl, D. M. Grant, K. G. Parker and T. L. Parker: Diamond Relat. Mater. 8 (1999) 457.

3 P. K. Chu, J. Y. Chen, L. P. Wang and N. Huang: Mater. Sci. Eng. Rep. 36 (2002) 143.

4 M. I. Jones, I. R. McColl, D. M. Grant, K. G. Parker and T. L. Parker: J. Biomed. Mater. Res. 52 (2000) 413.

5 F. Z. Cui and D. J. Li: Surf. Coat. Technol. 131 (2000) 481.

6 L. J. Yu, X. Wang, X. H. Wang and X. H. Liu: Surf. Coat. Technol. 128 (2000) 484.

7 D. A. LaVan, R. F. Padera, T. A. Friedmann, J. P. Sullivan, R. Langer and D. S. Kohane: Biomaterials 26 (2005) 465.

8 A.-S. Loir, F. Garrelie, C. Donnet, J.-L. Subtil, M. Belin, B. Forest, F. Rogemond and P. Laporte: Appl. Surf. Sci. 247 (2005) 225.

9 D. B. Chrisey and G. K. Hubler: Pulsed Laser Deposition of Thin Films (John Wiley \& Sons Inc., New York, 1994).

10 H. H. Tong, O. R. Monteiro, R. A. MacGill, I. G. Brown, G. F. Yin and J. M. Luo: Curr. Appl. Phys. 1 (2001) 197.

11 D. H. Kaelble and J. Moacanin: Polymer 18 (1997) 475.

12 S. Prawer, K. W. Nugert, Y. Lifshitz, G. D. Lempert, E. Grossman, J. Kulk, I. Avigal and R. Kalish: Diamond Relat. Mater. 5 (1996) 433.

13 Y. Liu, Z. Y. Li, Z. H. He, D. H. Chen and S. R. Pan: Surf. Coat. Technol. 201 (2007) 6851.

14 D. H. Chen, A. X. Wei and Z. Y. Li: Appl. Phys. A 80 (2005) 1573.

15 J. J. Hauser: J. Non-Cryst. Solids 23 (1977) 21.

16 P. Baurschmidt and M. Schaldach: Med. Biol. Eng. Comput. 18 (1980) 496.

17 N. Bark, Z. Foldes-Papp and R. Rigler: Biochem. Biophys. Res. Commun. 260 (1999) 35.

18 Y. X. Leng, J. Y. Chen, P. Yang, H. Sun, G. J. Wan and N. Huang: Surf. Sci. 531 (2003) 177.

19 T. I. T. Okpalugo, A. A. Ogwu, P. D. Maguire and J. A. D. McLaughlin: Biomaterials 25 (2004) 239.

20 D. J. Lyman, K. Knutson, B. Meneill and K. Shibatani: Trans. Am. Soc. Artif. Intern. Organ. 21 (1975) 49.

21 A. S. Hoffiman: Ann. N.Y. Acad. Sci. 516 (1987) 96.

22 S. M. Slack and T. A. Horbet: J. Biomater. Sci. Polym. 2 (1991) 227.

23 V. I. Sevastianov: CRC Crit. Rev. Biocompatibility 4 (1988) 109. 